Bundesgesundheitsbl 2015 $\cdot 58: 513-514$

DOI 10.1007/s00103-015-2154-1

Online publiziert: 28. März 2015

๑) Springer-Verlag Berlin Heidelberg 2015

\section{Oliver Razum ${ }^{1}$ - Anke-Christine Saß ${ }^{2}$}

${ }^{1}$ AG Epidemiologie \& International Public Health, Fakultät für Gesundheitswissenschaften, Universität Bielefeld, Bielefeld, Deutschland

${ }^{2}$ FG 27 Gesundheitsberichterstattung, Robert Koch-Institut, Berlin, Deutschland

\title{
Migration und Gesundheit: Interkulturelle Öffnung bleibt eine Herausforderung
}

Deutschland ist ein Einwanderungsland. Unter den rund 81 Mio. Menschen, die in Deutschland leben, haben laut dem Ausländerzentralregister 7,6 Mio. (9,4\%) eine ausländische Staatsangehörigkeit. Darunter sind die 1,5 Mio. Türkinnen und Türken die größte einzelne Nationalitätengruppe. Die Nationalität gibt aber nur einen unvollständigen Eindruck vom Migrationsgeschehen: Rund 20,5\% der Bevölkerung Deutschlands haben einen Migrationshintergrund, so das Statistische Bundesamt. Das heißt: 16,5 Mio. Menschen sind entweder selbst zugewandert oder sind Kinder von Zuwanderern.

Menschen mit Migrationshintergrund sind eine heterogene Gruppe, nicht nur im Hinblick auf ihr Herkunftsland, ihre Muttersprache und Kultur. Auch bezüglich gesundheitlicher Belastungen gibt es sehr unterschiedliche Befunde. Während neu Zugewanderte oftmals einen besonders guten Gesundheitszustand aufweisen (der so genannte „Healthy Migrant Effect"), können ungünstige Arbeitsbedingungen und eine benachteiligte sozioökonomische Situation mit zunehmender Aufenthaltsdauer zu einer Verschlechterung der gesundheitlichen Situation führen. Menschen mit Migrationshintergrund unterliegen also den gleichen sozioökonomischen Determinanten von Gesundheit wie die nicht migrierte Mehrheitsbevölkerung. Sie können jedoch in ihrem Lebenslauf anderen Risiken ausgesetzt gewesen sein - eine Betrachtung im Sinne der „Life-course“-Epidemiologie trägt dem Rechnung. Zudem gibt es Gruppen, die in besonderem Maße benachteiligt sind, weil sie keinen vollständigen oder geregelten Zugang zur Gesund- heitsversorgung haben. Dazu gehören die Roma in verschiedenen europäischen Ländern. Ebenso betrifft das Flüchtlinge, viele Asylbewerberinnen und Asylbewerber und Menschen ohne Aufenthaltsgenehmigung (sog. ,irreguläre“ Migrantinnen und Migranten) in Deutschland ein unhaltbarer Zustand, wie nun endlich auch die Politik erkennt [1].

Daten zur Gesundheit und Gesundheitsversorgung von Menschen mit Migrationshintergrund liegen aus verschiedenen Quellen vor. Jedoch fehlt es bislang an der routinemäßigen Berücksichtigung dieser großen Bevölkerungsgruppe in amtlichen Statistiken und Surveys. Auf diese Defizite wurde bereits im Schwerpunktbericht „Migration und Gesundheit" hingewiesen, der im Jahr 2008 in der Gesundheitsberichterstattung des Bundes erschien [2]. Auch heute noch wird in amtlichen Daten wie der Todesursachenstatistik - wenn das Merkmal überhaupt erfasst wird - oft nur nach deutscher und ausländischer Nationalität unterschieden. Mit den vorhandenen Daten lassen sich nur begrenzt Aussagen machen, selbst zur Sterblichkeit der Migrantinnen und Migranten im Vergleich zur Mehrheitsbevölkerung, wie der Artikel von Kohls zeigt. Ausgeprägt sind die Datendefizite auch im Bereich der psychiatrischen Versorgung, siehe den Beitrag von Schouler-Ocak et al. Umso wichtiger ist es, dass Menschen mit Migrationshintergrund in den vom Robert Koch-Institut durchgeführten Gesundheitssurveys angemessen berücksichtigt werden. Der Artikel von $\mathrm{Saß}$ et al. zeigt auf, in welchem Umfang Migrantinnen und Migranten an diesen Surveys teilgenommen haben.

\section{\) Gesundheitsbezogene Interventionen müssen stärker kultursensibel gestaltet werden}

Eine weiterhin hoch aktuelle Frage für Wissenschaft und Praxis ist, ob die Gesundheit der Menschen mit Migrationshintergrund primär von sozialen Determinanten abhängt; in der Mehrheitsbevölkerung ist dieser Zusammenhang inzwischen sehr gut belegt. Alternativ oder zusätzlich könnte sie von migrantenspezifischen Faktoren oder nicht angemessenem Zugang zur Versorgung bestimmt sein. Rommel et al. stellen anhand von Daten der „Studie zur Gesundheit Erwachsener in Deutschland“ (DEGS1) fest, dass nach der Kontrolle des sozioökonomischen Status Auswirkungen des Migrationshintergrunds auf die Gesundheit erkennbar bleiben. Ähnliche Ergebnisse fanden Brzoska \& Razum bei der Ergebnisqualität der medizinischen Rehabilitation. Migrationsspezifische Aspekte finden sich auch in der psychiatrischen Versorgung (Schouler-Ocak et al.) sowie bezüglich der Häufigkeit meldepflichtiger Infektionskrankheiten (Santos-Hövener et al.). Bei der Versorgung schwangerer Frauen in Großstädten mit hohem Migrantenanteil an der Bevölkerung scheint es hingegen kaum noch Defizite in der Versorgung zu geben, wie Brenne et al. aufzeigen.

$\mathrm{Zu}$ den besonderen Herausforderungen gehört die Frage, wie die Menschen mit Migrationshintergrund stärker an den verschiedenen Aspekten der gesundheitlichen Versorgung beteiligt werden kön- 
nen. Horn et al. beleuchten die Bedeutung von Health Literacy in der Beratung von Patientinnen und Patienten mit Migrationshintergrund. Brand et al. sowie Blümel stellen Aktivitäten und Initiativen im Bereich der Prävention und der gesundheitlichen Aufklärung vor. Habermann beschreibt abschließend die Entwicklung von Indikatoren für ein Integrationsmonitoring am Beispiel der Altenhilfe.

Wo Gesundheitsdienste und Einrichtungen noch nicht interkulturell geöffnet sind, können Menschen mit Migrationshintergrund nicht nur schlechter teilhaben. Selbst wenn sie Zugang zu den Diensten erhalten, so erzielen sie oft eine schlechtere Ergebnisqualität als die Mehrheitsbevölkerung. Gesundheitsbezogene Interventionen und Gesundheitsdienste müssen noch stärker kultursensibel gestaltet werden. Hingegen scheint es wenig zielführend, Dienste spezifisch für einzelne Gruppen von Menschen mit Migrationshintergrund anzubieten. Erforderlich ist vielmehr ein Diversity Management, das Bedarfe aller Menschen - unabhängig von ihrem Migrationshintergrund - weitmöglich berücksichtigt. Auch bei der Datenerhebung sind weitere Anstrengungen nötig, um möglichst alle Statistiken und Datenquellen zur Gesundheit interkulturell auszurichten. Aus den Daten abgeleitete „Taten“ sollten dann nicht nur kultursensibel, sondern auch evidenzbasiert sein. Bei der Evidenzbasierung bestehen allerdings noch Defizite, oftmals basiert (präventives) Handeln „nur“ auf Good Practice.

Migrantinnen und Migranten, aber auch Flüchtlinge und Asylsuchende sind oft junge und besonders aktive Menschen. Sie haben viele Wagnisse auf sich genommen, um nach Deutschland zu kommen und hier einen privaten und beruflichen Neuanfang zu gestalten. Für unsere alternde Gesellschaft spielt Migration eine wichtige Rolle. Zuwanderern gute Chancen für Beschäftigung, Bildung und Gesundheit zu bieten, sind wichtige Ziele einer gelingenden Integration. Bei der gesundheitlichen Versorgung der Migrantinnen und Migranten wurden in den vergangenen Jahren einige Verbesserungen erzielt - aber es bleibt noch vieles zu tun. Bei Asylsuchenden und Flüchtlingen kann und muss Deutschland allerdings deutlich mehr tun als bisher.

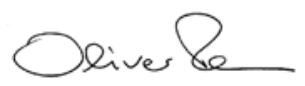

Prof. Oliver Razum

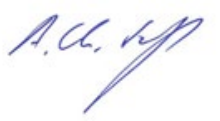

Dr. Anke-Christine Saß

\section{Korrespondenzadressen}

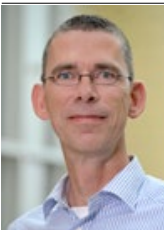

Prof. O. Razum

AG Epidemiologie \& International Public Health, Fakultät für Gesundheitswissenschaften Universität Bielefeld Universitätsstraße 25 33615 Bielefeld oliver.razum@uni-bielefeld.de

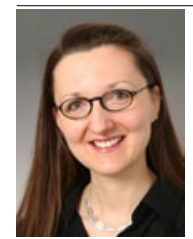

Dr. A. Christine Saß

FG 27 Gesundheitsberichterstattung Robert Koch-Institut General-Pape-Str. 62 12101 Berlin SassA@rki.de

\section{Literatur}

1. Beauftragte der Bundesregierung (2014) 10 . Bericht der Beauftragten der Bundesregierung für Migration, Flüchtlinge und Integration über die Lage der Ausländerinnen und Ausländer in Deutschland (Oktober 2014). Beauftragte der Bundesregierung, Berlin

2. Razum O, Zeeb H, Meesmann U, Schenk L, Bredehorst M, Brzoska P et al (2008) Migration und Gesundheit. Schwerpunktbericht der Gesundheitsberichterstattung des Bundes. Robert Koch-Institut, Berlin 